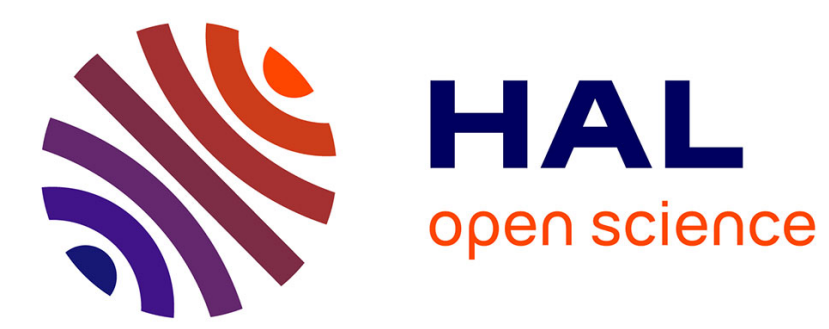

\title{
COMPUTER SIMULATION OF DISLOCATIONS
}

\author{
Rodolphe Perrin
}

\section{To cite this version:}

Rodolphe Perrin. COMPUTER SIMULATION OF DISLOCATIONS. Journal de Physique Colloques, 1974, 35 (C7), pp.C7-103-C7-111. 10.1051/jphyscol:1974710 . jpa-00215866

\section{HAL Id: jpa-00215866 https://hal.science/jpa-00215866}

Submitted on 1 Jan 1974

HAL is a multi-disciplinary open access archive for the deposit and dissemination of scientific research documents, whether they are published or not. The documents may come from teaching and research institutions in France or abroad, or from public or private research centers.
L'archive ouverte pluridisciplinaire HAL, est destinée au dépôt et à la diffusion de documents scientifiques de niveau recherche, publiés ou non, émanant des établissements d'enseignement et de recherche français ou étrangers, des laboratoires publics ou privés. 


\title{
COMPUTER SIMULATION OF DISLOCATIONS
}

\author{
R. C. PERRIN
}

Theoretical Physics Division, A. E. R. E. Harwell, Didcot, Oxfordshire, England

\begin{abstract}
Résumé. - L'auteur donne un aperçu des progrès récents obtenus dans la simulation sur ordinateur des dislocations dans un réseau cristallin. En particulier, deux nouvelles méthodes commodes pour trouver la configuration d'équilibre des atomes sont décrites en détail. Le simple champ des déplacements de l'élasticité linéaire anisotrope utilisé habituellement pour connaître les positions atomiques sur l'interface de séparation de la région atomique ne peut tenir compte d'aucun des effets non linéaires se produisant au cœur de la dislocation. Les traitements plus sophistiqués, nécessaires pour décrire l'effet à longue portée des non-linéarités de cour, sont discutés dans cette région d'interface élastique, y compris la dilatation qui accompagne la dislocation. On présente quelques résultats relatifs à la dissociation et de dislocations rectilignes, et de boucles, dans le cuivre.
\end{abstract}

\begin{abstract}
A review of recent advances in the techniques of computer simulation of dislocations in crystal lattices is given. Two efficient new methods of finding the equilibrium atomic configuration are described in detail. The simple linear anisotropic elastic displacement field which is normally used to determine atomic positions in the boundary surrounding the atomic region does not allow for any non-linear effects in the dislocation core. More sophisticated treatments of this elastic boundary region which can describe the long range effects of core non-linearities, including the dilatation associated with a dislocation, are discussed. Some results on the dissociation of both straight dislocations and loops in copper are presented.
\end{abstract}

1. Introduction. - The detailed atomic configurations of dislocation cores are important in determining many material properties. For example, the nature and degree of dissociation of the screw dislocation appears to be responsible for the asymmetry of slip in body centred cubic metals $[1,2]$ and the interaction between dislocations and point defects is critical in determining material behaviour in an irradiation environment. For instance, the considerable volume swelling of reactor materials irradiated at temperatures between one third and one half of their melting temperatures is attributed to the fact that dislocations have a stronger attraction for interstitial atoms than for vacancies [3].

The atomic configuration of crystal defects can best be obtained by direct computer simulation calculations in which the co-ordinates of a relatively small number of point atoms are stored in the computer. These atoms, which are arranged initially in some approximation to the defect to be investigated, are assumed to interact via some prescribed law (usually an axiallysymmetric two-body interaction) and the equilibrium configuration of the defect is obtained by finding the minimum energy configuration of this atomic assembly. The most important feature of such a calculation is obviously the choice of a realistic interaction between i] ( atc ms but since that subject has been considered c If r sively elsewhere [4-6] this paper will be devoted to two other important aspects of simulation calculations. In the following section efficient procedures for finding the equilibrium configuration are discussed and two new methods which are currently being used at Harwell with encouraging results are described.

In order to maintain stability of the atomic assembly it is necessary to surround it with a boundary region in which the atoms are held fixed during relaxation. Because of their long range strain fields the treatment of this boundary region is particularly important in the simulation of dislocations and recent developments in this area are described in the third section. In the final section some recent results on the dissociation of both straight dislocations and loops are presented.

2. Energy minimisation methods. - A considerable number of methods have been used by various authors to obtain the energy minimum and equilibrium configuration of the atomic assembly, the methods being based usually on computational simplicity rather than mathematical sophistication. Probably the most widely used method has been the so-called quenched dynamical method [7-9]. In this method, atoms which are not in equilibrium are allowed to accelerate under the influence of the net force upon them and their classical equations of motion are integrated by a simple finite difference scheme. A suitable choice of time step ensures that the kinetic energy of the assembly passes 
through a maximum after a few time steps and, since this corresponds to a local minimum of potential energy, the velocities of all the atoms are put to zero and the relaxation process is repeated. The method is simple to implement, has relatively modest storage requirements and seems better at avoiding unwanted metastable configurations than other methods.

An alternative approach has been to move each atom in turn to the position where the net force on it is zero and to cycle through each atom in the assembly many times until the equilibrium configuration is reached [10].

Two methods have been suggested by Sinclair and Pollard [11] which greatly reduce the number of evaluations of the interatomic interaction which are required but at the expense of inverting or diagonalising the matrix of second derivatives of the energy. As both inversion and diagonalisation of matrices are lengthy numerical procedures the value of these methods is restricted to those cases where repeated relaxations from similar starting configurations are required.

In discussing minimisation procedures it is useful to classify methods according to whether only the function to be minimised is evaluated or whether first and possibly second derivatives are also known. Methods which require the evaluation of derivatives will, in general, converge in many fewer steps and if the computational effort of calculating the derivatives is not excessive they are to be preferred. Because, near the minimum of the function, the second order terms in the Taylor series expansion dominate, minimisation methods have usually been constructed to have quadratic termination - that is, the exact minimum (to machine round-off error) of a quadratic function will be found in a finite number of steps. Specifically, the minimum of a quadratic function of $N$ variables will be found in at most $N^{2}$ steps if only the function is evaluated, in $N$ steps if first derivatives are known and if one step if the matrix of second derivatives is also known. Applied to more general functions the methods are iterative and convergence is not guaranteed in a finite number of steps but the quadratic termination feature should ensure rapid convergence as the minimum is approached. A minimisation method which makes use of second derivatives of the function is thus very efficient in terms of the number of function evaluations required which makes it particularly suitable for calculations where the evaluation of the interatomic interaction is lengthy, such as in ionic solids. However, the extensive storage requirements for the second derivative matrix make such a method unsuitable for a problem where the number of variables is large and the evaluation of the interatomic interaction is simple, which is usually the case for metals. Therefore, two methods are described below which have recently been implemented at Harwell. One is a second derivative method which is very efficient in terms of function evaluations while the other is a fast first derivative method with modest storage requirements.
2.1 VARIABLE METRIC METHODS. - In the method proposed by Sinclair and Pollard, based on inverting the matrix of second derivatives of the energy (the Hessian), they use the value of the inverse, calculated at the initial configuration, to speed the convergence of the method. This inverse will not be appropriate at the minimum, which will slow the rate of convergence. However, a class of methods, the so-called variable metric methods, do exist for updating an approximation to the inverse $\underline{\mathbf{H}}$ of the Hessian matrix $\mathbf{G}$ in such a way that as the minimum is approached the approximation $\underline{\mathbf{H}}$ tends to the true inverse $\mathbf{G}^{-1}$. The original variable metric method was due to Davidon [12] but the algorithm was simplified by Fletcher and Powell [13]. In the description of this method given below the following notation will be used :

$E$ : the crystal energy, is the function to be minimised.

$\mathbf{X}\left(x_{1}, x_{2}, \ldots, x_{N}\right)$ is the vector of co-ordinates of the atoms.

$\mathbf{g}$ : is the vector of derivatives of $E$ with elements $g_{i}=\partial E / \partial x_{i}$.

$\mathbf{G}$ : is the matrix of second derivatives of the energy (the Hessian) with elements $G_{i j}=\partial^{2} E / \partial x_{i} \partial x_{j}$.

$\underline{\mathbf{H}}$ : is an approximation to $\underline{\mathbf{G}}^{-1}$.

If $\underline{\mathbf{H}}_{0}$ is an approximation to $\underline{\mathrm{G}}_{0}^{-1}$ at the configuration denoted by $\mathbf{X}_{0}$ then, provided $\underline{\mathbf{H}}_{0}$ is positive definite, in analogy to the Newton-Raphson method a step in the direction $\mathbf{S}=-\underline{\mathbf{H}}_{0} \mathbf{g}_{0}$ will reduce the energy and give an improved approximation $\mathbf{X}_{1}$ to the equilibrium configuration.

If $\delta=\mathbf{X}_{1}-\mathbf{X}_{0}$ is the change in the configuration and $\gamma=\mathbf{g}_{1}-\mathbf{g}_{0}$ is the change in the vector of gradients, then an improved estimate of $\underline{\mathbf{H}}$ can be obtained from

$$
\underline{\mathbf{H}}_{1}=\underline{\mathbf{H}}_{0}+\frac{\delta \delta^{T}}{\delta^{T} \gamma}-\frac{\underline{\mathbf{H}}_{0} \gamma \gamma^{T} \underline{\mathbf{H}}_{0}}{\gamma^{T} \underline{\mathbf{H}}_{0} \gamma},
$$

where the superscript $T$ denotes transposition.

The alternative formula

$\underline{\mathbf{H}}_{1}=\underline{\mathbf{H}}_{0}-\frac{\delta \gamma^{T} \underline{\mathbf{H}}_{0}}{\delta^{T} \gamma}-\frac{\underline{\mathbf{H}}_{0} \gamma \delta^{T}}{\delta^{T} \gamma}+\left(1+\frac{\gamma^{T} \underline{\mathrm{H}}_{0} \gamma}{\delta^{T} \gamma}\right) \frac{\delta \delta^{T}}{\delta^{T} \gamma}$

which may deal better with difficult situations which might arise has been proposed by Broyden [14] and Fletcher [15]. In the original Fletcher Powell method the function $E$ was minimised in the direction $S$ before $\underline{\mathbf{H}}$ was updated and searching in a new direction was commenced. This linear minimisation allows a proof of quadratic convergence but requires several function evaluations. Fletcher [15] has proposed abandoning the linear search and taking just one step in the direction $\mathbf{S}$. He showed that provided the eigenvalues of $\underline{\mathbf{H}}$ tend monotonically to those of $\mathbf{G}^{-1}$, which is the case for both the above updating formulae (1) and (2), and a check was included to ensure the reduction of $E$ at each step, then ultimate convergence for quadratic functions 
was assured. Extensive testing [15] showed that this strategy was superior, in terms of function evaluations, to the Fletcher-Powell algorithm. This minimisation method has been incorporated by Norgett [16] in a Harwell program package HADES (Harwell Automatic Defect Evaluation System) for investigating the configurations of point defects in ionic solids. Results which have been obtained with this program indicate that the method is very efficient [17].

2.2 The CONJugate gradients method. - The need to store the inverse of the second derivative matrix makes the variable metric method, described above, impractical for use where large numbers of atoms must, of necessity, be included, such as in the determination of the dissociation of dislocations in materials of low stacking fault energy. A suitable method for such large calculations is the conjugate gradients method of Fletcher and Reeves [18], since it requires only the evaluation and storage of the first derivatives of the energy.

In this method, if $\mathbf{X}_{i}$ is an approximation to the equilibrium configuration, then an improved approximation $\mathbf{X}_{i+1}$ is generated by finding the minimum of the energy along a search direction $\mathbf{S}_{i}$. If the search directions are chosen to be mutually conjugate with respect to the matrix of second derivatives $\underline{\mathbf{G}}$, that is if

$$
\mathbf{S}_{i}^{T} \underline{\mathbf{G}} \mathbf{S}_{j}=0 \quad \text { for } i \neq j,
$$

then, for quadratic functions, once the gradient has been set to zero in a particular search direction it is not disturbed by minimising the gradient in another search direction. This ensures that the method is quadratically convergent. This conjugacy condition may be imposed without explicit evaluation of $\mathbf{G}$ by choosing the search direction $\mathbf{S}_{i}$ to be a linear combination of the gradient vector $\mathbf{g}_{i}$ and the previous search directions $\mathbf{S}_{j}(j=0$ to $i-1$ ), which satisfy the condition (3). If the initial search direction $\mathbf{S}_{0}$ is taken as the direction of steepest descent, then Fletcher and Reeves give

$$
\begin{aligned}
& \mathbf{S}_{0}=-\mathbf{g}_{0} \\
& \mathbf{S}_{i}=-\mathbf{g}_{i}+\frac{\mathbf{g}_{i}^{T} \mathbf{g}_{i}}{\mathbf{g}_{i-1}^{T} \mathbf{g}_{i-1}} \mathbf{S}_{i-1}
\end{aligned}
$$

as a simple formula for choosing search directions which satisfy the conjugacy condition. Alternative formulae for choosing $\mathbf{S}_{i}$ have been discussed by Fletcher [19].

The search direction having been chosen, the second part of the problem is to find the step length $\alpha$ which minimises the energy in that direction. For general functions it is not possible to solve this linear search problem exactly in a finite number of steps and an approximate solution must be accepted. A suitable criterion is that the gradient in the search direction is reduced by a prescribed amount, i. e.

$$
\left|\mathbf{g}^{T}\left(\mathbf{X}_{i}+\alpha \mathbf{S}_{i}\right) \mathbf{S}_{i} / \mathbf{g}^{T}\left(\mathbf{X}_{i}\right) \mathbf{S}_{i}\right| \leqslant \rho<1 .
$$

If the chosen step length $\alpha$ is such that the condition (5) is satisfied then a new search direction is chosen in accordance with (4) and the iteration is continued. If the choice of $\alpha$ fails to satisfy (5) then one of the following actions is taken. If $\alpha$ was too small

$$
\left(\mathbf{g}^{T}\left(\mathbf{x}_{i}+\alpha \mathbf{S}_{i}\right) \mathbf{S}_{i}<0\right)
$$

an additional step is taken with the step length calculated on the assumption that $\mathbf{g}^{T} \mathbf{S}_{i}$ varies linearly with $\alpha$. If $\alpha$ was too large $\left(\mathbf{g}^{T}\left(\mathbf{x}_{i}+\alpha \mathbf{S}_{i}\right) \mathbf{S}_{i}>0\right)$ it is revised using a cubic interpolation formula [18] and the step is repeated. The initial guess at $\alpha$ is based on the assumption that the reduction in $E$ on the current iteration will be equal to that on the previous iteration. Then if $E$ is assumed to be a quadratic function of $\alpha$ an excellent estimate is given by

$$
\alpha=2\left(E_{i-1}-E_{i}\right) / \mathbf{g}_{i}^{T} \mathbf{S}_{i} .
$$

With this choice of $\alpha$, the condition (5), with $\rho$ set at 0.1 , was usually satisfied with one interpolation or extrapolation.

This procedure has been incorporated in a Harwell program DEVIL (Defect EValuation In Lattices) for simulating defects in systems with relatively short ranged potentials, such as are typically used for metals. It has been used by Norgett, Perrin and Savino [20] and Perrin and Savino [21] to determine the dissociation of the edge and screw dislocations in copper and by Savino and Perrin [22] to describe the morphology of vacancy loops in copper. In the latter calculation over 5000 atoms (15000 variables) were included and the equilibrium configuration was obtained with about 150 evaluations of the interatomic interaction. This suggests an approximate rule that one function evaluation is required for every hundred variables, which is considerably better than is obtained with the quenched dynamical method.

3. Continuum boundaries around the atomic region. Since the region of crystal which may be simulated atomistically is very small (a few thousand atoms at most) the boundary conditions which are imposed upon this region are obviously important, especially for the simulation of dislocations because of their extremely long range strain field. To confirm with a notation in common use, the region of crystal in which the atoms are treated as freely interacting particles will be referred to as region $\mathrm{I}$. In the region surrounding this, termed region II, the atomic co-ordinates, which are required for the evaluation of the forces on atoms in region I, are derived from elasticity theory.

In early calculations of dislocation configurations $[23,24]$ the displacement fields from isotropic elasticity theory were used in region II. The potentials in current use have generally been fitted to, or reproduce satisfactorily, the observed elastic constants and so the simulation of the atomic region will reproduce the full anisotropy of the crystal. The use of boundary 
displacements derived from isotropic theory is, therefore, no longer justified in accurate calculations, especialiy as the anisotropic elastic displacements around a dislocation are readily available from the work of Eshelby, Read and Schockley [25]. Although the method of Eshelby et al. is well known the principal formulae are set out below to facilitate a later discussion of recent developments.

If we consider an infinite straight dislocation line lying along the $X_{3}$ axis, then the translational symmetry along the dislocation line means that the displacements, stresses, etc. can only be functions of the co-ordinates $X_{1}$ and $X_{2}$. Eshelby et al. show that the displacements can be written as

$$
u_{i}=\sum_{\alpha=1}^{3} A_{i}^{\alpha} f_{\alpha}\left(z_{\alpha}\right)
$$

where $f_{\alpha}$ is an arbitrary function of

$$
z_{\alpha}=X_{1}+p_{\alpha} X_{2}
$$

and the complex coefficients $p_{\alpha}$ and $A_{i}^{\alpha}$ are obtained by substituting the form (7) in the equilibrium equations. The fact that the stress associated with the dislocation must remain analytic means that $\mathrm{d} f_{\alpha} / \mathrm{d} z_{\alpha}$ is analytic and hence can be expanded in a Laurent series. This leads to $f_{\alpha}$ taking the form

$$
f_{\alpha}=\frac{D_{\alpha}}{2 \pi i} \ln z_{\alpha}+\sum_{n=-\infty}^{\infty} C_{n}^{\alpha} z_{\alpha}^{n} .
$$

The logarithmic term provides the required closure failure round a Burgers circuit and so the coefficients $D \alpha$ are determined by the dislocations Burgers vector and the fact that it is subject to no net body force. The other terms allow arbitrary boundary conditions to be satisfied on any given boundary.

A slightly simplified form of the Eshelby et al. theory has been given by Stroh [26] but a particularly useful formulation has been given by Willis [27]. In the Eshelby et al. and Stroh formulations it is necessary to solve a set of linear equations for the coefficients $A_{i}^{\alpha}$. When the $X_{1}-X_{2}$ plane is a plane of symmetry these equations uncouple, which simplifies the analytical treatment of the particular case, but makes the general case more difficult to program. The Willis approach is particularly suitable in this respect as it gives an explicit expression for the displacements.

In applications of the above theory to provide the displacements for boundary atoms in simulation calculations $[28,9]$ it has generally been assumed that it is sufficient to use the logarithmic term, which provides the long range field of the dislocation, and that, if the atomic region is large enough, the higher terms have decayed sufficiently to be neglected. This may require the use of a very large atomic region to avoid a mismatch of displacements at the boundary. This is particularly relevant to the simulation of dislocation dissociation where the atomic region required may be very large. In their simulation of the $\langle 110\rangle$ edge dislocation in copper, Norgett, Perrin and Savino [20] found that it was necessary to have 1120 atoms in the atomic region to avoid spurious boundary effects.

Sinclair [29] has shown how the higher terms in the expansion (9) may be included in determining the elastic displacements of the boundary and that inclusion of these terms allows the size of the atomistic region to be reduced considerably without loss of accuracy. He considered the particular case of the $<100>$ edge dislocation in a cubic crystal for which an analytical solution for the logarithmic term was available from the work of Eshelby et al. [25]. Since it is more convenient to compute with real quantities Sinclair re-wrote the expression for the displacements as

$$
\mathbf{u}=\mathbf{u}_{0}+\sum_{n=1}^{\infty} \sum_{j=1}^{4} S(n, j) \mathbf{U}(n, j)
$$

where $\mathbf{u}_{0}$ includes the usual logarithmic terms and the real coefficients $S(n, j)$ are related to the complex coefficients $C_{n}^{\alpha}$ of (9) by

$C_{n}^{(1)}=S(n, 1)+S(n, 2)+i S(n, 3)+i S(n, 4)$

$C_{n}^{(2)}=S(n, 1)-S(n, 2)+i S(n, 3)-i S(n, 4)$.

The index $\alpha$ takes only the values 1 and 2 as $u_{3}=0$. The higher order terms $U(n, j)$ are simple linear combinations of $z_{\alpha}^{-n}$. For instance

$$
\begin{aligned}
& U_{1}(n, 1)=\operatorname{Re}\left[z_{(1)}^{-n}+z_{(2)}^{-n}\right] \\
& U_{2}(n, 1)=\operatorname{Re}\left[-A\left(z_{(1)}^{-n}-z_{(2)}^{-n}\right)\right]
\end{aligned}
$$

where $A$ is a constant [29].

This re-arrangement makes some terms odd functions of $X_{1}$ (those with $n$ and $j$ either both even or both odd) so that their coefficients must be zero if the $X_{2}-X_{3}$ plane has mirror symmetry, which is the case for the cube edge dislocation.

The coefficients $S(n, j)$ were determined as follows. The atoms in region $I$ and the surrounding continuum region II were positioned according to the long range field $\mathbf{u}_{0}$ and then the atoms in region I were allowed to relax to their equilibrium configuration. If the potential describing the interaction between atoms in region I was used to examine the forces on those atoms in region II which were close enough to interact with region $I$, then these forces were in general significant, indicating that the continuum displacements were incorrect. The forces on these boundary atoms were reduced to zero by treating the coefficients $S(n, j)$ as generalised co-ordinates of the system and determining their conjugate forces $F_{\mathrm{s}}$

$$
\begin{aligned}
F_{s} & =-\frac{\partial F}{\partial S}=\sum_{k}-\nabla_{k} E \cdot \frac{\partial X_{k}}{\partial S} \\
& =\sum_{k} \mathbf{f}_{k} \cdot \mathbf{U}(n, j) .
\end{aligned}
$$

Here $\mathbf{f}_{k}$ is the net force on atom $k$, and $k$ is summed over those atoms in region II which can interact with 
region I atoms through the interatomic potential. The field coefficients $S(n, j)$ and the co-ordinates of the atoms in region I were then adjusted until the generalised forces $F_{s}$, as well as the forces on region I atoms, were zero.

In his calculation, Sinclair included these terms for $n$ up to 4 ( 8 non-zero field coefficients) and varied the size of region I from 100 to 276 atoms. He found that the variation of atomic bond lengths in the dislocation core with the size of region I was much less when these extra terms were included in the elastic solution. With the simple boundary due only to the long-range field, the maximum variation in bond length when the number of atoms in region I was increased from 100 to 276 was $1 \%$ and this was reduced to $0.04 \%$ by the inclusion of the higher terms.

An alternative approach to improving the simple linear elastic boundary solution and reducing the mismatch between region I and region II has been adopted by Gehlen, Hirth, Hoagland and Kanninen [30] who also considered the $\langle 100\rangle$ edge dislocation in b. c. c. iron. Their method is as follows. Atoms in both the atomic and boundary regions are given the linear isotropic elastic displacements appropriate to the cube edge dislocation and then the atoms in region $I$ are allowed to move towards their equilibrium positions using the quenched dynamical relaxation method. After a number of iterations of the relaxation procedure it is terminated, a cylindrical surface $\Gamma$, centred on the dislocation, line is drawn in the atomic region and the tractions on this surface are calculated. The force exterted on an atom on the outside of $\Gamma$ by an atom in the interior is assumed to act on the surface $\Gamma$ at the point where the line of action of the force intersects $\Gamma$. (It is necessary to subtract from this the force which would exist between the same atoms in the perfect lattice, otherwise this prescription would give non-zero tractions in the perfect lattice.) The surface $\Gamma$ is divided into a number of equal segments and the resultant force per unit area is resolved into normal and tangential components. The distribution of these tractions around $\Gamma$ is conveniently expressed by fitting to a Fourier series. Knowledge of these tractions on $\Gamma$, together with the assumption of zero applied stress on the external surface, allows the displacements in the continuum region to be recalculated. Relaxation of region $I$ is continued, with the above adjustments being made to region II, until the tractions on $\Gamma$ are consistent with the displacements in both regions.

By matching the additional elastic fields introduced by this procedure to the fields produced by a pair of double forces, Gehlen et al. concluded that the extra fields could be well described by an elliptical dilatation source, the centre of which was displaced from the centre of the dislocation towards the region of tension by a distance of $5.2 \AA$.

In agreement with the conclusions of Sinclair [29] they found that the more sophisticated treatment of the displacements in region II allowed the number of atoms in region I to be reduced by a factor of 2 or 3 without loss of accuracy. The detailed configuration of the dislocation core was very similar to that obtained by Sinclair in spite of the use of isotropic elasticity in the boundary. This is illustrated in table I where a comparison of a typical interatomic bond length in the dislocation core is displayed for a number of models considered by both authors.

The small variation of bond length with model size when the improved boundaries are used in striking, as is the agreement on its magnitude. These results also suggest that, even with 780 atoms in region $\mathrm{I}$, the simple fixed boundary is having an influence on the core configuration. A more detailed comparison of results is given in reference [30].

These extra terms in the elastic boundary have their origin in the non-linear response of the interatomic potential used in region I and are therefore capable of describing the volume change associated with the creation of a dislocation. This volume change was not evaluated by Sinclair but Gehlen et al. quote a value of $0.25 b^{2}$ per unit length of dislocation, although this value is not very well converged, being rather sensitive to the model size.

Sinclair and Bullough [31] have investigated the effect of including in region II the non-linear elastic displacement field derived by a second order perturbation method due to Willis [32]. In general, the improvement in the displacement field produced by this second order solution was not sufficient to justify the added complexity of the calculation. An exception, however, was the $\langle 100\rangle$ screw dislocation in the body centred cubic lattice (not a dislocation of practical interest) where a worthwhile improvement over the linear solution was found. The reason for this is that,

TABLE I

\begin{tabular}{|c|c|c|c|c|c|}
\hline $\begin{array}{l}\text { Atoms in } \\
\text { Region I }\end{array}$ & $\begin{array}{l}\text { Sinclair } \\
\text { Boundary }\end{array}$ & $\begin{array}{c}\text { Bond } \\
\text { Length } \AA\end{array}$ & $\begin{array}{l}\text { Atoms in } \\
\text { Region I }\end{array}$ & $\begin{array}{l}\text { ehlen et al. } \\
\text { Boundary }\end{array}$ & $\begin{array}{c}\text { Bond } \\
\text { Length } \AA\end{array}$ \\
\hline$\overline{100}$ & Improved & $4 . \overline{391} 8$ & $\overline{100}$ & Improved & 4.3964 \\
\hline 276 & Improved & 4.3900 & 340 & Improved & 4.3925 \\
\hline 100 & Simple & 4.3181 & 340 & $(*)$ & 4.3915 \\
\hline 276 & Simple & 4.3599 & 780 & Simple & 4.3839 \\
\hline
\end{tabular}

(*) With approximate correction for anisotropy, 
for a screw dislocation perpendicular to a plane of elastic symmetry, the second order perturbation solution is fortuitously accurate to third order. Even in this case they found that the higher orde1 terms in the linear solution were more important than the inclusion of non-linear effects. They concluded that a better solution of the non-linear equations than the second order perturbation treatment of Willis [32] was required.

In an atomic simulation of edge dislocations in ionic crystals, Granzer, Belzner, Bücher, Petrasch and Teodosiu [33] have used a non-linear displacement field which is the exact third order elastic solution, but no details of the method were given.

\section{Dissociation of dislocations in the face-centred} cubic lattice. - 4.1 Strarght $\frac{1}{2}<110>$ DISLOCATION. - In the face-centred cubic lattice, a dislocation with a $\frac{1}{2}<110>$ Burgers vector can lower its energy by dissociating on a (111) plane into a pair of Shockley partial dislocations separated by a region of intrinsic stacking fault.

For example

$$
\frac{1}{2}[\overline{1} 10] \rightarrow \frac{1}{6}[\overline{1} 2 \overline{1}]+\frac{1}{6}[\overline{2} 11] .
$$

In a recent computer simulation of the edge and screw dislocations in copper, Norgett, Perrin and Savino [20] found that this dissociation did occur. In these calculations the cubic spline potential constructed by Englert, Tompa and Bullough [34] was used to simulate the interaction between the atoms. This empirical potential was fitted to the experimental elastic constants and in the repulsive core region it was matched to a BornMayer potential which reproduced the experimental displacement energies. It was scaled to give a vacancy formation energy of $1.1 \mathrm{eV}$ and a stacking fault energy of $70 \mathrm{ergs} \mathrm{cm}^{-2}$. That this potential predicts a reasonable fault energy for copper is particularly important as this, together with the mutual repulsion of the partials, determines the extent of the dissociation.

The equilibrium separation of the partials was determined by first giving the atoms in both the inner and boundary regions the anisotropic elastic displacements corresponding to two partial dislocations with an arbitrary separation on the slip plane. Then, with the boundary region held fixed, the inner region was allowed to relax to its equilibrium configuration using the conjugate gradients method described above. When equilibrium was attained the displacements, from perfect lattice positions, of the (111) planes on either side of the slip plane were computed. The centres of the partials were taken to be the points where the relative displacements of these planes in the [110] direction were equal to $\frac{1}{4}$ and $\frac{3}{4}$ of the total Burgers vector. The elastic displacements appropriate to this separation of the partials were then reimposed on the assembly and the inner region was allowed to relax as before. This procedure was continued iteratively and the final equilibrium configuration was achieved when the elastic displacements on the boundary atoms were those appropriate to the separation of the partials in the relaxed inner region. The elastic displacements imposed on the boundary atoms were the long range anisotropic elastic displacements derived from the logarithmic terms in (9). With 1120 atoms included in the inner region it was found that the displacements in the atomic region matched smoothly on to the elastic displacements in the boundary.

The equilibrium atomic configurations of the edge and screw dislocations, which are shown in figures 1 and 2 as projections on the $\{111\}$ slip plane, proved to

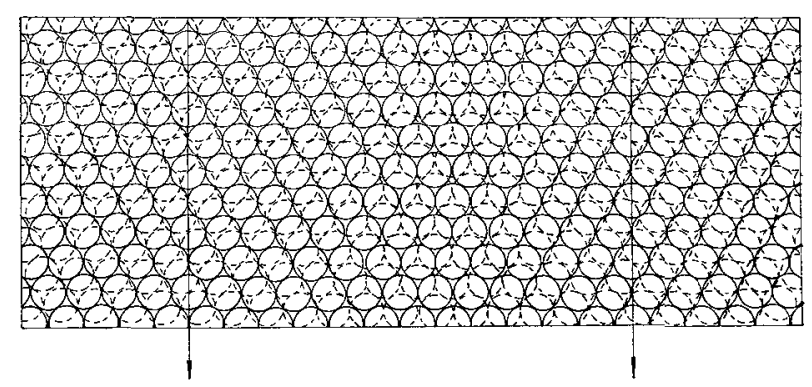

FIG. 1. - A $\{111\}$ projection of the atomic configuration, across the slip plane, of the edge dislocation in copper. The position of the partials is indicated.

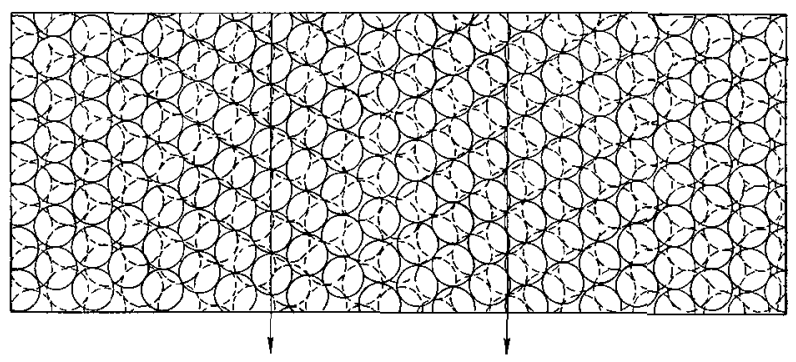

FIG. 2. - A $\{111\}$ projection of the atomic configuration, across the slip plane, of the screw dislocation in copper. The position of the partials is indicated.

be very different from the equivalent configurations derived from elasticity theory. The separation of the partial dislocations was $32 \AA(13 b$, where $b$ is the total Burgers vector) for the edge dislocation and $15 \AA(6 b)$ for the screw orientation which are to be compared with the separations of $8 b$ and $2.5 b$ predicted by anisotropic elasticity theory. Also, the Peierls widths of the partials were much greater than would be expected on the basis of elasticity theory. (The width of a partial was defined as that distance over which the relative displacement across the slip plane was within $\pm \frac{1}{8} b$ of the value at the centre of the partial.) For example, the partial width of $2 b$ derived from elasticity was increased to $8 b$ in the edge orientation. These wide 
partials leave only a comparatively small region, approximately $5 \mathrm{~b}$ wide, which can reasonably be described as a perfect stacking fault. This can be seen quite clearly by examining figure 1. In fact, these atomic simulatioǹn models suggest that for such small separations of the partials it is better to think of the relative displacement across the glide plane as a continuous distribution of infinitesimal dislocations rather than two discrete cores separated by a perfect fault.

The extent of dissociation derived from the simulation calculation is in reasonably good agreement with recent electron microscope observations using the weak beam imaging technique. For example, the calculated dissociation of $32 \AA$ for the edge dislocation agrees with the measured value of $38 \AA$ quoted by Stobbs and Sworn [35] and Cockayne, Jenkins and Ray [36] within the experimental error limits. An exact comparison of these results is not possible since elasticity theory was used in deriving the partial separation from the observed images. The most striking agreement, however, is that the simulation calculations predict a ratio of $2.2: 1$ for the variation in the extent of dissociation in going from the edge to the screw orientation. This is in close agreement with the observations of Stobbs and Sworn who found a value of $2.1: 1$ whereas anisotropic elasticity theory would predict that it should be $3.2: 1$. This agreement is encouraging as it is likely that any systematic error in deducing the partial separations from the microscope image will be much smaller for this ratio than for the absolute values of the dissociation.

To obviate the necessity of using elasticity theory to interpret the experimental images, Perrin and Savino [21] calculated weak beam electron microscope images directly from the atomic configuration of the edge dislocation. Figure 3 shows the images they
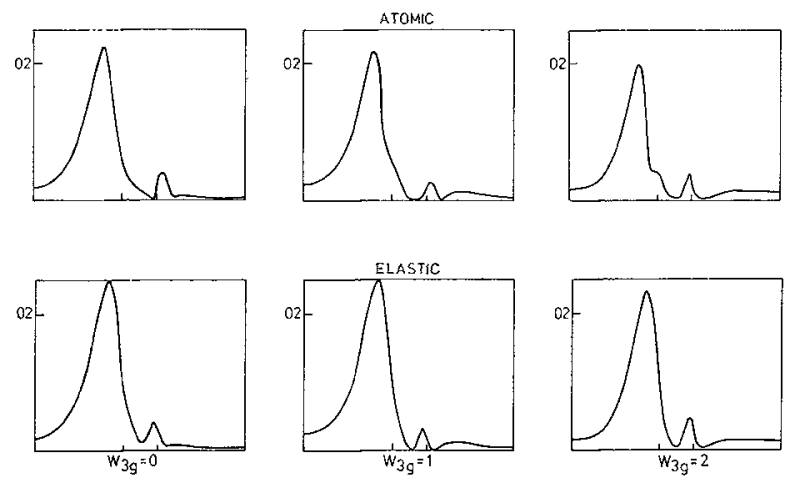

FIO. 3. - The intensity profiles of $\mathbf{g} \cdot \mathbf{b}=2$ weak beam images of the dissociated edge dislocation in copper for $w_{3 \mathrm{~g}}=0,1,2$. The top row has been calculated from the atomic model and the bottom row from elasticity for the same partial separation of $32 \AA$.

obtained from the atomic configuration using the weak [220] reflection with the [6 60$]$ reflection set at the Bragg condition and at deviations $w_{3 g}=1$ and 2. Also shown in the same figure are the images obtained using the anisotropic elastic displacement field for the same separation of the partials. From this figure it can be seen that the intensity peaks in the images do not correspond to the positions of the partials and that the displacement is considerably greater for the atomic model.

The results they obtained from the simulation model for the extent of dissociation and the separation of the intensity peaks in the weak beam images are summarised in table II, together with the results obtained from anisotropic elasticity theory for the same stacking fault energy. The third row of the table shows how the observed separation of the intensity peaks in the image of $47 \AA$ leads to a predicted stacking fault energy of 41 ergs $\mathrm{cm}^{-2}$ when analysed on the basis of elasticity theory. Since the image peak separation of $54 \AA$ obtained from the computer simulation calculation agreed with the observed value, within the experimental uncertainty, Perrin and Savino concluded that analysis of the weak beam images by the simulation model using the Englert potential would lead to a value of the intrinsic stacking fault energy in copper slightly greater than $70 \mathrm{ergs} \mathrm{cm}^{-2}$ rather than the value of $41 \mathrm{ergs} \mathrm{cm}^{-2}$ obtained by Stobbs and Sworn and Cockayne et al.

\section{TABLE II}

$\begin{array}{cccc}\text { Model } & \begin{array}{c}\text { Fault energy } \\ \text { erg cm }\end{array} & \begin{array}{c}\text { Partial } \\ \text { Separation }\end{array} & \begin{array}{c}\text { Peak } \\ \text { Separation }\end{array} \\ - & - & - & \AA \\ \text { Atomic } & 70 & 32 & - \\ \text { Elastic } & 70 & 20 & 54 \\ \text { Elastic } & 41 & 38 & 47\end{array}$

This technique of calculating weak-beam electron microscope images from the atomic configurations of dislocations obtained from computer simulation calculations promises to give one of the most direct links between this type of calculation and experimental observations.

4.2 FRANK LOOPs. - The complex nature of the electron microscope images of vacancy loops formed by ion bombardment has led to the suggestion that the dislocation bounding these loops is not the simple $\frac{1}{3}$ [111] Frank dislocation but that some form of dissociation has taken place [37-39]. The efficient conjugate gradients method of minimisation has made it feasible to simulate regions of crystal large enough (over 5000 atoms) to study the morphology of such loops and Savino and Perrin [22] have recently carried out a simulation of small planar vacancy clusters in copper in order to determine the nature of the bounding dislocation. The Englert et al. potential described above was used to define the interaction between the atoms. The defects which they considered were regular triangular and hexagonal loops with $\langle 110\rangle$ edges 
lying on a close-packed (111) plane. The triangular loop which contained 36 vacancies had sides $20 \AA$ long while the hexagonal loop containing 37 vacancies was $18 \AA$ in diameter.

They found that the Frank dislocations bounding the triangular loop dissociated, on the (111) planes passing through the edges of the loop, into a stair rod dislocation and a Shockley partial

$$
\frac{1}{3}[111] \rightarrow \frac{1}{6}[110]+\frac{1}{6}[112] \text {. }
$$

The Shockley partials on adjacent (111) planes interacted to produce a stacking fault tetrahedron by the mechanism described by Silcox and Hirsch [40]. There was, however, a small amount of unfaulting at each corner of the tetrahedron. This can be seen in figure 4 which shows a projection on the face of the

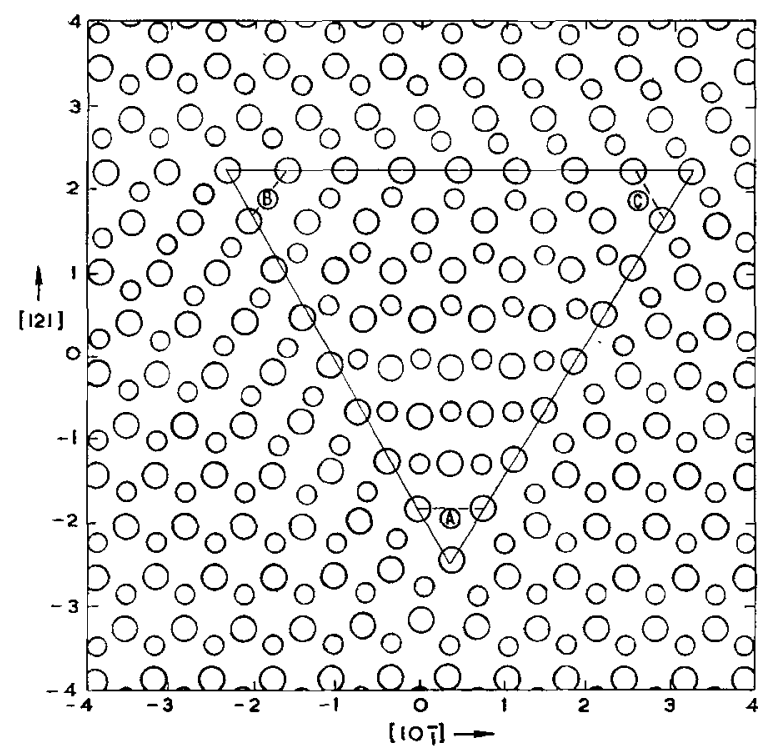

FIG. 4. - The atomic configuration across one of the faces of a stacking fault tetrahedron in copper. The small circles represent atomic positions inside the face of the tetrahedron while the larger circles are just outside. The atom at $A$ has unfaulted, $B$ has partially unfaulted and $C$ has remained faulted.

tetrahedron of the (111) planes of atoms immediately inside (small circles) and outside (large circles) this face. Clearly, the atom at $C$ is in the almost perfectly faulted position, the atom at $A$ has unfaulted to near its perfect lattice position, while the atom at $B$ is in an intermediate position. (Compare the relative positions of the atoms with the perfect lattice in the corner of the figure.) A similar unfaulting occurs on each of the faces but from a different corner of the tetrahedron on each face.

For the hexagonal loop the principal relaxations were found to take place on the (111) planes which pass through the edges of the loop, the relaxation being on opposite sides of the loop plane for alternate edges of the hexagon. As shown in figure $5 a$, the intersection of these planes defines a rhomboid enclosing the original loop. Again the Frank dislocations dissociated into stair rods and Shockley partials but because of the more complex configuration associated with the hexagonal loops the Shockley partials were unable to react together to form stair rods as they did for the triangular loop and so they did not pass completely across the faces of the rhomboid. This can be seen from figure $5 b$ where contours are plotted of the relative

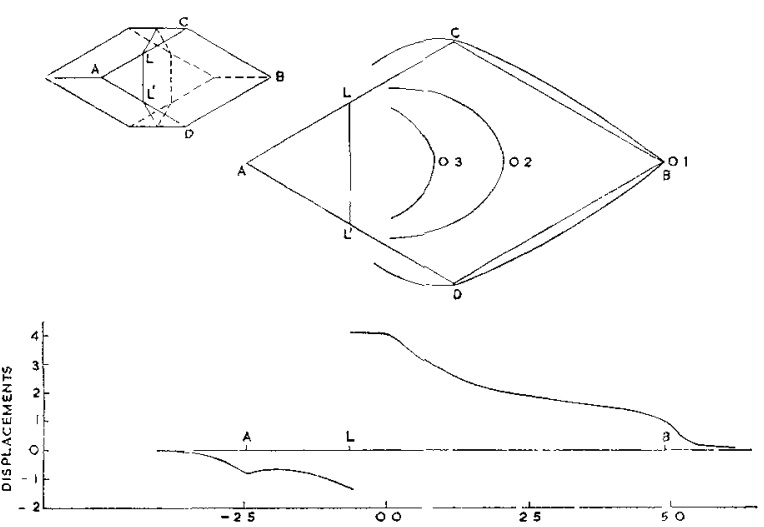

FIG. 5. - The dissociation of a hexagonal vacancy loop in copper. a) The thomboid formed by the $\{111\}$ planes passing through the edge of the hexagonal loop. $b$ ) Contours of the relative displacement of atoms, in the $\langle 112\rangle$ direction $A B$, across the face $A C B D$. c) The displacements of (b) plotted along the line $A B$ (all distances are in units of the lattice parameter).

displacement in the $\langle 112\rangle$ direction $A B$ of the planes on either side of the face $A B C D$. Since the complete passage of a $\frac{1}{6}<112>$ partial causes a relative displacement of 0.41 (in units of the lattice parameter) the 0.2 contour may be thought of as the centre of a rather wide Shockley dislocation. This extreme width can be clearly seen in figure $5 c$ where the $<112>$ displacement is plotted along the line $A B$. The displacement can be seen to approach the full Shockley partial value of 0.41 at the loop $\mathrm{L}$ while on the other side of the loop the displacement of 0.14 corresponds to the projection of the $\frac{1}{6}\langle 110\rangle$ stair rod on to the $<112>$ direction.

\section{References}

[1] Vitek, V., Perrin, R. C. and Bowen, D. K., Phil. Mag. 21 (1970) 1049.

[2] Basinski, Z. S., Duesbery, M. S. and TAYlor, R., in «Interatomic Potentials and Simulation of Lattice Defects », p. 537 (1972). Ed. by Gehlen, P. C., Beeler, J. R. Jr, and Jaffee, R. I. (Plenum Press).
[3] See for example Bullough, R. and Perrin, R. C. in «Voids Formed by Irradiation of Reactor Materials 》, p. 79 (1971). Ed. by Pugh, S. F., Loretto, M. H. and Norris, D. I. R. (British Nuclear Energy Society).

[4] Torrens, I. M., Interatomic Potentials (Academic Press, N. Y.) 1972. 
[5] Johnson, R. A., J. Phys. F : Metal Physics 3 (1973) 295. [6] Preceeding papers at this conference.

[7] Gibson, J. B., Goland, A. N., Milgram, M. and VineYARD, G. H., Phys. Rev. 120 (1960) 1229.

[8] Bullough, R. and Perrin, R. C., Proc. R. Soc. A 305 (1968) 541.

[9] Gehlen, P. C., Rosenfield, A. R. and Hahn. G. T., J. Appl. Phys. 39 (1968) 5246.

[10] Johnson, R. A. and Brown, E., Phys. Rev. 127 (1962) 446.

[11] Sinclair, J. E. and Pollard, H. F., Phys, Lett. 32A (1970) 93.

[12] Davidon, W. C., Argonne Nat. Lab. Rep. ANL-5990 (Rev).

[13] Fletcher, R. and Powell, M. J. D., Comput. J. 6 (1963) 163.

[14] BRoYden, C. G., J. Inst. Maths. App. 6 (1970) 222.

[15] Fletcher, R., Comput. J. 13 (1970) 317.

[16] NorgetT, M. J., Harwell Report A. E. R. E.-R. 7650 (1974).

[17] Lidiard, A. B. and Norgett, M. J., in "Computational Solid State Physics », p. 385 (1972). Ed. by Herman, F., Dalton, N. W. and Koehler, T. R. (Plenum Press).

[18] Fletcher, R. and Reeves, C. M., Comput. J. 7 (1964) 149.

[19] Fletcher, R., Harwell Report A. E. R. E.-R. 7073 (1972).

[20] Norgett, M. J., Perrin, R. C. and Savino, E. J., J. Phys. F : Metal Physics 2 (1972) L 73.

[21] Perrin, R. C. and Savino, E. J., J. Micros. 98 (1973) 214.

[22] Savino, E. J. and Perrin, R. C., J. Phys. F : Metal Physics 4 (1974) 1889.
[23] Huntingdon, H. B., Dickey, J. E. and Thompson, R., Phys, Rev. 100 (1955) 1117.

[24] Cotterill, R. M. J. and Doyama, M., Phys. Rev. 145 (1966) 465.

[25] Eshelby, J. D., Read, W. T. and Shockley, W., Acta Met. 1 (1953) 251.

[26] Stroh, A. N., Phil. Mag. 3 (1958) 625.

[27] Wrllis, J. R., Phil. Mag. 21 (1970) 931.

[28] Bullough, R. and Perrin, R. C. in «Dislocation Dynamics », p. 175 (1968). Ed. by Rosenfield, A. R., Hahn, G. T. and Bement, A. L.

[29] Sinclatr, J. E., J. Appl. Phys. 42 (1971) 5321.

[30] Gehlen, P. C., Hirth, J. P., Hoagland, R. G. and KanNINEN, M. F., J. Appl. Phys. 43 (1972) 3921.

[31] Sinclair, J. E. and Bullough, R. To be published.

[32] Wrulus, J. R., Int. J. Engng. Sci. 5 (1967) 171.

[33] Ganzer, F., Belzner, V., Búcher, M., Petrasch, P. and Teodosiv, C., J. Physique Colloq. 34 (1973) C9-359.

[34] Englert, A., Tompa, H. and Bullough, R. in "Fundamental Aspects of Dislocation Theory », Nat. Bur. of Standards Special Publn. 317 (1970) 273.

[35] Stobis, W. M. and Sworn, C. H., Phil. Mag. 24 (1971) 1365.

[36] Cockayne, D. J. H., Jenkins, M. L. and Ray, I. L. F., Phil. Mag. 24 (1971) 1383.

[37] Häussermann, F., Phil. Mag. 25 (1972) 537.

[38] Wilson, M. M. and Hirsch, P. B., Phil. Mag. 25 (1972) 983.

[39] Jenkins, M. L., Phil. Mag. 29 (1974) 813.

[40] Silcox, J. and Hirsch, P. B., Phil. Mag. 4 (1959) 72. 